\title{
Isolation and expression of UB05, a Plasmodium falciparum antigen recognised by antibodies from semi-immune adults in a high transmission endemic area of the Cameroonian rainforest
}

\author{
Vincent Pryde Kehdinga Titanji*, Alfred \\ Amambua-Ngwa, Damian Nota Anong, Stanley \\ Kimbeng Mbandi, Emily Tangie, Ivo Tening \\ and Raymond Yengo
}

Department of Biochemistry and Microbiology, Faculty of Science, University of Buea, Buea, Cameroon

\begin{abstract}
Background: Antibodies in adults living in malaria endemic areas that target specific parasite antigens are implicated in protective immunity to infection and disease. This study aimed to identify, isolate and characterise targets of protective immunity in malaria. A Plasmodium falciparum antigen termed UB05 (Genbank Accession Number DQ235690: PlasmoDB PF10_ 0372) that had been isolated by immunoscreening with semi-immune sera was studied.

Methods: Polymerase chain reaction, sequencing and bioinformatics were used to analyse the UB05 gene. A specific mouse anti-UB05 antibody was used in parasite reinvasion growth/inhibition assays and in immunoflourescence to localise the antigen. In a crosssectional study, enzyme linked immunosorbent assay was used to study immunoglobulin $\mathrm{G}(\mathrm{lg} \mathrm{G})$ responses to the antigen.

Results: The gene revealed significant homologies with gene sequences from Plasmodia and other apicomplexan parasites and had two alleles in the wild $P$. falciparum isolates. The antigen is expressed by schizonts and segmented merozoites. Mouse antibodies against it marginally inhibit in vitro invasion of erythrocytes by $P$. falciparum. The $\lg G$ responses to UB05 were found to be significantly lower $(p<0.05)$ in the sera of children (2-5 years) compared with adults ( $>18$ years), with or without parasitaemia. However, parasitaemia correlated inversely $(r=0.7-$ 0.75) with serum anti-UB05 IgG concentrations. Furthermore, anti-UB05 lgG concentrations were lower in the sera of febrile patients (body temperature $>37.5^{\circ} \mathrm{C}$ ) than their non-febrile counterparts regardless of parasitaemia status.
\end{abstract}

*Corresponding author: Professor Vincent P.K. Titanji, Biotechnology Unit, University of Buea, P.O. Box 63, Buea, South West Province, Cameroon

Phone: + 237 3323134, Fax: + 2373322272 ,

E-mail: vpk.titanji@yahoo.com

Received December 2, 2008; accepted June 19, 2009
Conclusions: These results are compatible with a role for UB05 in the development of immunity and as a marker of protective immunity to malaria. Clin Chem Lab Med 2009;47:1147-58.

Keywords: antigen; fever; immuno-screening; malaria; Plasmodium falciparum; vaccine.

\section{Introduction}

Malaria, a parasitic disease caused by protozoa of the genus Plasmodium ranks second of the ten top causes of death in Africa (1). It kills 1-3 million children worldwide every year; $90 \%$ of these fatalities occur among African children. A three-pronged approach has been recommended for fighting the disease, namely, rapid detection followed by combination therapy; the use of mosquito bed nets and targeted mosquito destruction $(2,3)$. However, the emergence and spread of drug and insecticide resistances has posed serious challenges to these strategies. Hence, efforts to develop a malaria vaccine remain high on the international scientific agenda (4). Clinical, epidemiological and experimental evidence strongly support the feasibility of a malaria vaccine (5). However, it has been difficult to construct a sub-unit malaria vaccine from the leading malaria vaccine candidates that are currently under intensive investigation $(6,7)$. It is possible that all the critical components required for an effective and long-lasting sub-unit vaccine have not yet been identified. In fact, although the $P$. falciparum genome codes for nearly 5300 structural genes, only $40 \%$ of these genes are known (8). Conceivably, additional protective antigens remain to be identified among the unknown gene products. Since systematic testing of all possible candidates is not technically feasible, it is necessary to devise approaches for short-listing the candidates that may contribute to protective immunity. One such approach is differential immuno-screening using sera from semiimmune and susceptible subjects $(9,10)$. Antibody transfer experiments suggest that immunoglobulin $\mathrm{G}$ (lgG) antibodies are critical effectors of protective immunity to malaria $(11,12)$. Indeed, many of the malaria vaccines under investigation are designed to elicit protective antibodies $(7,13)$.

Herein, we describe the identification using differential immuno-screening and molecular cloning of a dominant antigen termed UB05 (GenBank Accession Number DQ235690) that was previously listed as a hypothetical protein (PlasmoDB PF10_0372) of unknown function on chromosome 10 of the $P$. falciparum 
genome. We show that UB05 is preferentially recognised by IgG antibodies from semi-immune adults, and suggest that it may be a marker of immune protection against malaria.

\section{Materials and methods}

\section{Study sites}

Most of the studies were carried out at Bolifamba, a small multi-ethnic trading and farming village of 3500 inhabitants on the western flank of Mount Cameroon (14). Additional blood samples were collected at the Kumba Medical Research station located $\sim 150 \mathrm{~km}$ north of Bolifamba.

\section{Study population}

Ethical clearance for human population studies was obtained from the South West Regional Delegation of Public Health. The study population was comprised of adult volunteers and children who came to the Malaria Pilot Centre Bolifamba for consultation, or in response to a general announcement through the quarter heads in the markets and churches. The aims of the project were explained to the participants and informed consent was sought and received from all volunteers or their guardians in the cases of minors. Upon recruitment, participants were clinically examined and blood samples were collected and used for the diagnosis of malaria following previously described standard procedures (14, 15).

\section{Immuno-screening}

A P. falciparum 3D7 cDNA expression library (UNI-ZAP XR) from MR4 (www.mr4.org) was screened using pooled antisera from selected sub-population of participants. The endemic groups whose sera were used for gene library screening had been described as susceptible or semiimmune (15). They comprised 23 infected children (IC) (1-5 years) and 25 semi-immune adults ( 18 years or older). The semi-immune adults included those who had lived in the high transmission malaria endemic community for at least 5 years, had no reported case of clinical malaria and were shown by microscopy to remain persistently parasite free for over 12 months without any chemoprophylaxis. Sera from these individuals were prepared from whole blood by centrifugation. Pooled high-titre sera for each group were depleted of anti-Escherichia coli antibodies using E. coli total proteins coupled to $\mathrm{CNBr}$ activated Sepharose-4B following the manufacturer's instructions. Prior to screening, the titre of $P$. falciparum cDNA library was determined by serially diluting (10-fold) in SM buffer and plating in order to obtain a dilution that avoided overlapping plaque formation on the agar plates. E. coli strain XL-1 Blue MRF (Stratagene, La Jolla, California, USA) was infected with the diluted phage cDNA library, followed by an overnight incubation at $37^{\circ} \mathrm{C}$ or $42^{\circ} \mathrm{C}$ for $\sim 2 \mathrm{~h}$ until pinpoint size plaques were visible. The library was screened using 1:100 dilutions of sera from IC and partially immune adults following established protocols (16). Positive plaques specific to partially immune adults were re-screened at a lower density. This process was repeated three times until populations of only positive plaques were obtained.

\section{PCR amplification}

Single plaques containing recombinant clones were selected and transferred to sterile tubes with $100 \mu \mathrm{L}$ of SM buffer
(100 mM NaCl, $50 \mathrm{mM}$ Tris- $\mathrm{HCl}$ at $\mathrm{pH}$ 7.5; $10 \mathrm{mM} \mathrm{MgSO}_{4}$ ). The agar plugs were incubated in $\mathrm{SM}$ buffer overnight at $4^{\circ} \mathrm{C}$ before being used as templates in the individual PCRs. The cDNA inserts were amplified using PCR in $50 \mu \mathrm{L}$ reactions using the vector-specific primers T3 and T7, as described previously (17). All PCR reaction products were separated using $1.5 \%$ agarose gels containing ethidium bromide to verify purity and determine their size. Amplicons were purified using the Sephaglas ${ }^{\circledR}$ bandprep kit (Pharmacia, Uppsala, Sweden). Each DNA band was eluted with $30 \mu \mathrm{L}$ of distilled $\mathrm{H}_{2} \mathrm{O}$. For long-term storage, a drop of chloroform was added to the SM buffer containing pure positive clones.

\section{DNA sequencing and sequence analysis}

Freshly purified PCR products of each clone were subcloned into pCRIITopo-TA vectors as described by the manufacturer (Invitrogen, Carlsbad, CA, USA). Each cloning reaction was $6 \mu \mathrm{L}$ in total volume and included $3 \mu \mathrm{L}$ PCR product, $1 \mu \mathrm{L}$ $200 \mathrm{mM} \mathrm{NaCl} ; 10 \mathrm{mM} \mathrm{MgCl}, 1 \mu \mathrm{L}$ sterile $\mathrm{dH}_{2} \mathrm{O}$ and $1 \mu \mathrm{L}$ vector. Reaction mixtures were used to directly transform $50 \mu \mathrm{L}$. Top 10 cells made chemically competent by washing in cold $100 \mathrm{mM} \mathrm{CaCl}_{2}$ and storing overnight at $4^{\circ} \mathrm{C}$. Transformed cells were selected by growing on ampicillin plates and then screened for the presence of inserts by PCR using T3 and T7 primers and/or the M13 forward and reverse primers. Positive clones were grown overnight in LB medium and plasmids prepared using Wizard DNA preparation kit (Promega, Stockholm, Sweden). Plasmid preparations were checked for purity using 1\% agarose and two separate preparations for each cDNA clone were sequenced commercially by MWG Biotech (Ebersberg, Germany).

\section{Bioinformatics}

Nucleic acid sequences of clones derived with the M13reverse primer were transformed into the reverse complement strand prior to analysis. After editing with VecSreen National Centre for Biotechnology Information (NCBI) to filter out vector fragments, the nucleic acid sequences were compared with public databases (GenBank non-redundant nucleotide and protein databases) using the BLAST family of algorithms with default parameters $(18,19)$. The open reading frames were determined by open reading frame (ORF)Finder and conserved-domain searches were performed using NCBI conserved domain search $(20,21)$. The ORF sequences of each clone were also directly analysed against protein databases at Swisprot and trEMBL. Significant matches were grouped and analysed for genetic distance using ClustalW, Phylip and SplitsTree programmes. Homologous sequences were also examined for the number and length of matching motifs, along with conserved alignment positions by ClustalW. Predictions of the physicochemical and structural properties of the derived protein sequences were performed using the protein analysis software available at Expasy

\section{PCR analysis of field isolates}

To further characterise one of the clones, UB05, specific primers were designed against the ORF of the target sequence and used to amplify DNA from malaria-infected blood with PCR. They included: UB05FP-GGCATCCTTCCTTTATATGTT and UB05RP-TTATGTGCGTCATCTTTACCT, with an empirically determined optimum annealing temperature of $60^{\circ} \mathrm{C}$. The specificity of the primers was checked by performing a blast search against public DNA databases. DNA samples that were analysed were extracted from 68 infected 
blood samples collected from patients living in the malaria endemic rural community of Bolifamba, and from 80 infected school children around Kumba, Cameroon. DNA templates for the nested PCR assay were prepared from the whole blood spot using the simple Chelex-100 (Sigma-Aldrich, St. Louis, MO, USA) boiling method. Each spot was boiled in $180 \mu \mathrm{L}$ of Chelex-100 solution and $\sim 120 \mu \mathrm{L}$ of DNA template was obtained. Five microliter of this was used in the nested PCR assay.

Prior to UB05 specific PCR, the species of Plasmodium in each sample was determined using nested PCR following a previously described protocol $(22,23)$. PCR products were analysed on $1.5 \%$ agarose gels containing ethidium bromide. Amplicons were cut-out, purified, cloned into Topo-TA and sequenced as described above.

\section{Expression constructs}

For cloning into prokaryotic expression vectors, the UB05 ORF was amplified directly from isolated phages with specific primers spanning the entire ORF, or made to exclude 75 nucleotides at the $5^{\prime}$-end coding for the first $25 \mathrm{~N}$-terminal amino acids. These products were cloned directly into TOPO expression vectors, pBAD thioTOPO-TA, or pEXP5-NT. The complete or truncated ORF was also amplified with primers engineered to have restriction enzyme sites for cloning into pET32a, pETM11 and pETM-30 E. coli expression vectors. All recombinant constructs were first amplified in Top10 cells against appropriate antibiotics. Plasmids, purified as above, were checked by sequencing before being used for transformation of expression hosts. Bacteria E. coli host strains tested for expression included Novablue, Top10, BL21, BL21 (DE3)pLys and BLR. Induction conditions with either IPTG or arabinose were determined empirically and applied as appropriate.

\section{Expression and purification of recombinant UB05}

For high-level expression of UB05 from pEXP5NT, plasmids were freshly transformed into the appropriate $E$. coli strain (BL21(DE3)pLys). A single colony was inoculated into $20 \mathrm{~mL}$ of LB medium with the recommended antibiotic and incubated overnight on a shaker at $37^{\circ} \mathrm{C}$. On the following day, $10 \mathrm{~mL}$ of the overnight culture was inoculated in $1 \mathrm{~L}$ of fresh LB or 2YT supplemented with antibiotics. The cultures were grown on a shaker at $37^{\circ} \mathrm{C}$ until the $\mathrm{OD}_{600}$ was at least 0.9 . After inducing with $1 \mathrm{mM}$ IPTG, cells were further cultured at $42^{\circ} \mathrm{C}$ or $37^{\circ} \mathrm{C}$ in a shaker for $4 \mathrm{~h}$, or overnight, respectively. Induced cells were collected following centrifugation at $4000 \mathrm{~g}, 4^{\circ} \mathrm{C}$, for $10 \mathrm{~min}$. Fractions of wet induced cell pellets were resuspended in phosphate buffer, pH 7.4 $(20 \mathrm{mM}$ sodium phosphate buffer and $500 \mathrm{mM} \mathrm{NaCl}$ with $10 \mathrm{mM}$ imidazole) or $50 \mathrm{mM}$ Tris buffer at $\mathrm{pH}$ 7.3. These buffers were supplemented with $6 \mathrm{M}$ urea in case of denaturing purification. To minimise protein degradation, all manipulations were done in a refrigerated chromatography cabinet. Cells were lysed using several rounds of sonication. After each sonication the suspension was centrifuged at $9000 \mathrm{~g}, 4^{\circ} \mathrm{C}$, for $30 \mathrm{~min}$. Supernatants that were collected were pooled, treated with benzonase nuclease (Novagen, Merck, Nottingham, UK) and filtered through a $0.45-\mu \mathrm{m}$ filter before use in protein purification. Recombinant proteins were purified by affinity chromatography ( $1 \mathrm{~mL}$ HiTrap chelating HP columns, Amersham Biosciences, Uppsala, Sweden). The recombinant proteins were eluted in $100 \mathrm{mM}$ phosphate buffer, $\mathrm{pH}$ 7.4 with $500 \mathrm{mM}$ imidazole (Sigma-Aldrich, St. Louis, MO). Proteins were further purified with Sephadex G-75 gel filtration chromatography on an AKTA-FPLC system (Amersham
Biosciences). All protein expressions and purification steps were checked using sodium dodecyl sulphate-polyacrylamide gel electrophoresis (SDS-PAGE).

\section{Plaque purification of UB05 plaque specific antibodies}

High titre UB05 phages were used to infect XL-1 blue cells and grown in top SOB agarose on LB plates. Phages were grown at $37^{\circ} \mathrm{C}$ to pinpoint plaques and then overlaid with nitrocellulose discs impregnated with $10 \mathrm{mM}$ IPTG. After an hour of incubation, filters were lifted and washed thrice for $10 \mathrm{~min}$ each in phosphate-buffered saline (PBS)-tween. Filters were then blocked with $1 \%$ gelatine and washed in PBS to remove excess blocking agent. Using a clean Petri dish, filters were overlaid with $3 \mathrm{~mL}$ of a 1:40 dilution of depleted partially immune human serum pool and incubated overnight at $4^{\circ} \mathrm{C}$ with intermittent rocking. The following day, unbound antibodies were washed off with five changes of PBS-tween and then with PBS. Next, antibodies were eluted with rocking filters in three rounds of $1 \mathrm{~mL}$ volumes of $150 \mathrm{mM}$ glycine- $\mathrm{HCl}, \mathrm{pH}$ 2.5. The antibody eluates were immediately equilibrated and stabilised by adding four volumes of $0.6 \mathrm{mg} / \mathrm{mL}$ bovine serum albumin (BSA) in $1.5 \mathrm{M}$ Tris buffer, $\mathrm{pH}$ 8. They were stored diluted 1:1 in glycerol at $-20^{\circ} \mathrm{C}$ until needed.

\section{Immunisation of mice with UB05}

Polyclonal antisera were raised in BALB/c mice that were immunised on days 0,21 , and 30 with an emulsion containing $\sim 50 \mu \mathrm{g}$ of purified UB05 fusion recombinant protein in PBS - incomplete Freund's adjuvant (IFA) (1:1) at two sites subcutaneously. Blood was collected prior to initial immunisation and after the last boost from the tail vein. The serum fraction was assayed for UB05 specific antibody content using a standard enzyme-linked immunosorbent assay (ELISA) as described previously $(15,16)$.

\section{SDS-PAGE and Western blot analysis}

SDS-PAGE and Western blotting were performed according to standard methods as described elsewhere $(23,24)$. Plasmodium antigens from infected blood cultures, recombinant bacteria cell extracts and purified recombinant proteins were separated using either $5 \%-20 \%$ gradient or $12.5 \%$ SDS-polyacrylamide gels, and then analysed by staining with Coomassie Brilliant Blue or by immunoblotting on nitrocellulose. Antibodies used for blotting included plaque purified UB05 human antibodies, anti-6His monoclonal antibodies (Pharmacia), mouse anti-UB05 antibodies and human sera. Optimum antibody or sera dilutions for immunoblotting were determined empirically for each assay. Bound antibodies were revealed by reaction with rabbit antihuman-lgG or goat anti-mouse horse raddish peroxidise conjugates (Sigma) and diaminobenzidine (Sigma).

\section{Localisation of UB05 antigen}

An indirect immunofluorescent antibody (IFA) test to detect the location of the UB05 protein in $P$. falciparum was performed on air dried films of infected red blood cell (RBC) as described previously $(25,26)$. Briefly, P. falciparum strain F32 parasite culture, enriched for late stage schizonts by flotation on $1 \%$ gelatine, were used. Mature parasite cultures were spotted onto slides and fixed in methanol or acetone. They were then incubated with different dilutions of purified antiUB05 lgG for $1 \mathrm{~h}$ at room temperature. Labelled chicken anti- 
mouse (Alexa Flour) and goat anti-chicken antibodies (Molecular Probe, USA) diluted 1:500 in Tris Hanks (TH) were used as secondary and tertiary antibodies, respectively. Between incubations, the slides were washed 3 times with PBS. The end-point titre was taken as the highest dilution which gave specific staining against the parasites.

\section{Growth inhibition studies}

We compared the reinvasion/growth inhibition activity of the mouse antisera to UB05, purified mouse anti-UB05 lgG and mouse pre-immune IgG using a modified protocol previously established $(25,26)$. The IgG fraction from the pre-immune and immune sera was purified using Ab SpinTrap ${ }^{T M}$ affinity chromatography. Briefly, target uninfected $\mathrm{O}^{+}$erythrocytes were washed three times in RPMI and used directly or labelled with fluorescein isothiocynate (FITC). Labelled cells were washed an additional 3 times in RPMI 1640 to remove all unincorporated FITC. Invasion assays were set with synchronous rings or trophozoites $P$. falciparum strains 3D7 and F32 at 2\% parasitaemia. Triplicate assays of each decreasing doubling dilutions of test antibodies and malaria culture medium were incubated in a candle jar at $37^{\circ} \mathrm{C}$, with a change of medium every $24 \mathrm{~h}$. After $60 \mathrm{~h}$, the erythrocytes were washed with PBS and then stained with ethidium bromide or hydroethidine fluorescent vital stain to detect parasite DNA. A total of 50,000 stained cells/assay were then counted by flow cytometry (FACS Calibur, BD bioscience, Franklin Lakes, NJ, USA). Percent inhibition by antibodies was determined as [1-(proportion of target cells invaded in presence of UB05 antibodies/proportion of target cells invaded in pre-immune antibodies) $] \times 100$.

\section{Protein determination}

Protein concentrations in the chromatographic fractions were estimated at $280 \mathrm{~nm}$ using an Ultrospec 1000 spectrophotometer (Pharmacia Biotech). In all other experiments, protein quantities were measured using standard Bradford assay with bovine serum albumin as a standard.

\section{Detection of antibodies to clone UB05 in human sera}

Sera samples from $P$. falciparum infected and uninfected individuals in the Bolifamba endemic community were assayed using ELISA for concentrations of UB05 specific IgG. Five $\mu \mathrm{g} / \mathrm{mL}$ of the purified recombinant protein was used in these assays. Antisera from patients were assayed at a dilution of 1:100 in PBS containing $5 \%$ non-fat milk. The nonparametric Mann-Whitney test was employed to determine the significance of differences in antibody levels in different patient groups at a $95 \%$ confidence limit.

\section{Results}

\section{Differential screening of $\boldsymbol{P}$. falciparum 3D7 library}

In the primary screening experiments, a dilution of $1: 150,000$ of the phage library stock in SM buffer was used. This dilution gave at least 2000 pfu per plate for each filter. Twenty-thousand clones were screened in ten primary screens. The anti-E. coli antibody-depleted healthy adult (HA) and IC sera employed in screening were highly reactive against crude Plasmodium antigens using ELISA (Figure 1). A total of 63 clones were identified by the HA pool, 37 by the IC pool and
23 by both serum pools. Twenty-six distinct clones were picked at random from the HA clones, and ten from the IC clones for further rounds of screening. After the fourth round of screening, 11 clones were distinctly recognised only by the HA pool, and two, coded IC 1 and IC 2 were recognised by the IC pool. These clones remained positive on further rounds of screening. The HA serum pool recognised 5 times more $P$. falciparum antigens than the ICs pool. Thus, nine clones preferentially recognised by the HA pool and two by the IC pool were purified for sequencing and further analysis.

\section{Nucleic acid sequence analysis}

Eleven isolated clones that were sequenced were arbitrarily code-named serially from UB01 to UB11. UB stands for University of Buea. Following their nucleic acid sequence analyses, the HA specific clones comprised three groups of well-aligned sequences: group I - UB01, UB05, UB06, UB07 and UB11; group II - UB08 and UB09, and group III - UB03 and UB10 (Figure 2). Blastn results against the $P$. falciparum database at NCBI confirmed the Plasmodium origin of the clones of groups I and II. They aligned to a regions of $P$. falciparum chromosomes with group I (UB05) matching to a region of chromosome 10 predicted to code for a hypothetical protein PF10_0372. Genomic sequences of the UB05 gene from laboratory and wild isolates were 1062-1262 bp long. The entire gene is comprised of four exons and three introns containing varying numbers of TTTA, GTAT, TATA and TTTTTA repeats. Group II sequences showed at least $97 \%$ homology to $P$. falciparum derived nucleic acid entries including mRNA for exported antigen Ag 5.1 (PFAG51ER), a P. falciparum circumsporozoite protein-related antigen (M11145.1) and $P$. falciparum export protein EXP-1 (X05074.1). Sequences obtained for group III were truncated fragments of Plasmodium DNA that were not studied further.

\section{Deduced amino acid sequence analyses}

The longest cDNA sequences for each group above; UB05 (474 bp), UB09 (378 bp) and UB10 (214 bp),

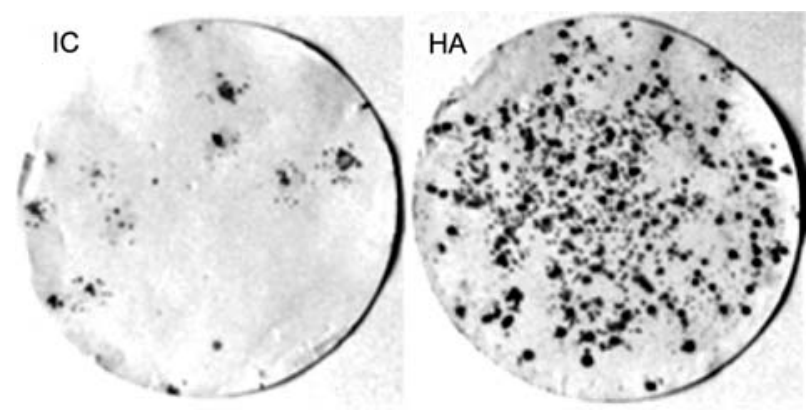

Figure 1 Typical plague lifts from $P$. falciparum (3D7) expression library in lambda zap vector.

The nitrocellulose lifts were probed with infected children sera (IC) and healthy adult (HA, semi-immune) sera from a high transmission malaria zone. Other experimental details are given under Materials and methods. 


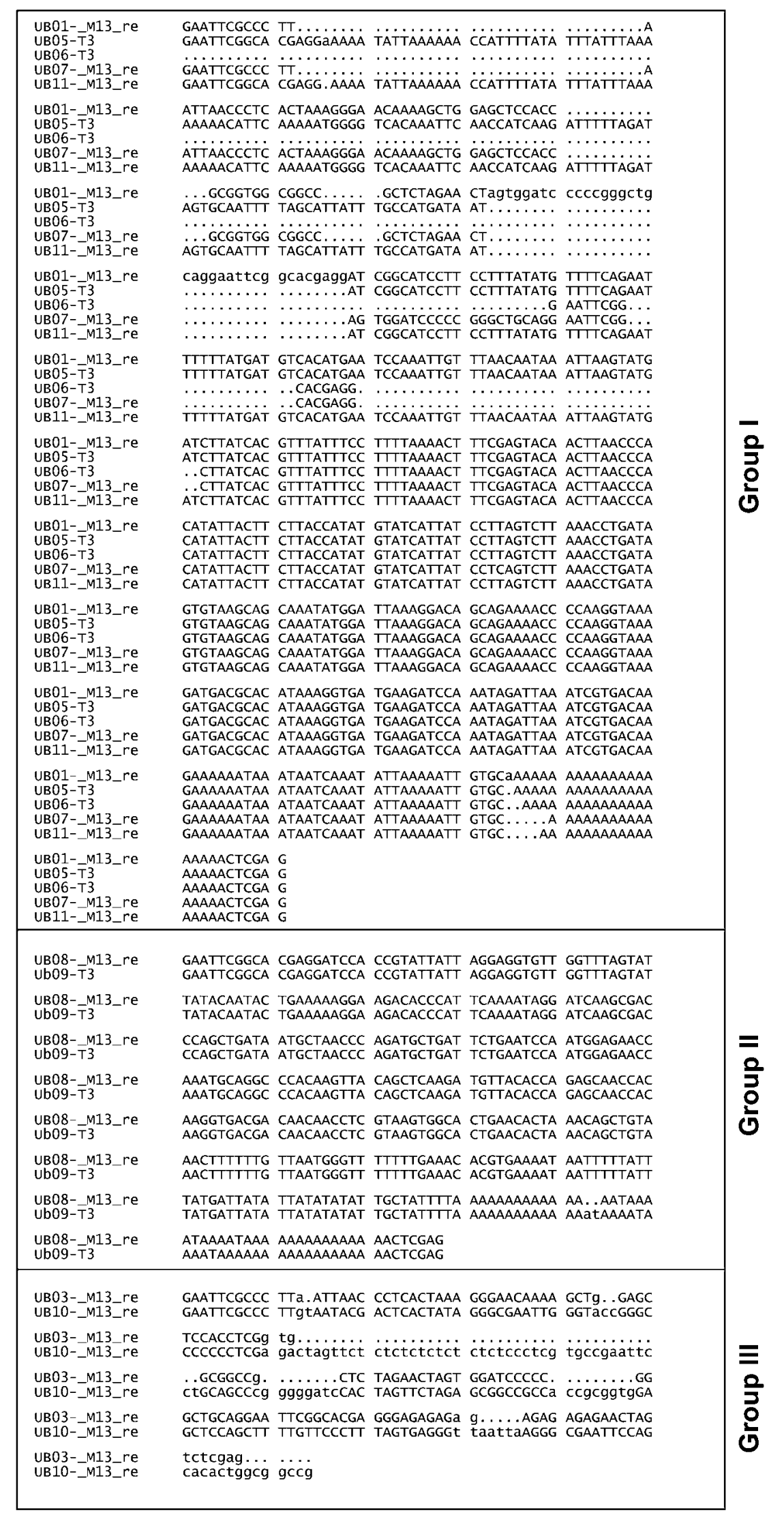

Figure 2 Multiple sequence alignments of cDNA of clones UB01-_M13_re (455 bp), UB03-_M13_re (142 bp), UB05-T3 (474 bp), UB06-T3 (272 bp), UB07-_M13_re (366 bp), UB11-_M13_re (470 bp), UB10-_M13_re (214 bp), and UB08-_M13_re (376 bp) Ub09T3 (378 bp). 
were selected for further derived protein sequence analyses. Blastp and tBlastx results for UB09 confirmed the nucleic acid alignment results as found for the derived ORF. It showed $97 \%$ homology to a fragment of circumsporozoite-related antigen and $99 \%$ to export protein-I (EXP-I). UB10 translated into a 56-aa peptide fragment homologous to the C-terminal of Plasmodium berghei strain ANKA hypothetical protein (PB108231.00.0). This protein belongs to the class of unconventional myosins found in all Plasmodia and other organisms. The UB05 deduced amino acid sequence was homologous to 14 other protein sequences entries in databases. The most significant matches comprised derived apicomplexan ORFs from Plasmodium spp. (PF10_0372, PB000898.01.0, PC000827.00.0, PY04877), Clostridium spp. (cgd2_ 1690, Chro.20183), Theileria spp. (TP04_0076, TA07565), Toxoplasma spp. and Bebesia spp. genomes or ESTs, while two statistically insignificant matches were to fragments of yeast (TIM21) and mycoplasmal proteins (mhp478). The amino acid sequences of eight homologues are aligned in Figure 3. The sequences are highly conserved between species within each of the protozoa genera. Some amino acid positions were conserved absolutely or by biochemical character between all eight species. They included particularly (numbering for UB05) RFLD (aa9-12), F19, L28, L31, E33, RLFPF (54-58) and from N64 to D83. The Plasmodium spp. sequences had a conserved RDKKK (116-119). Except for the sequence from $P$. yoelli yoelli that was truncated ( 30 amino acids), all the sequences were predicted by SignalP and Psort programmes at high probabilities to have an uncleavable $\mathrm{N}$-termi- nal leader/signal peptide at least 25 amino acids long, otherwise described as a signal anchor. Other predicted conserved features included two transmembrane helices sandwiching an absolutely conserved RLFPFK loop, two or three C-terminal protein kinase C phosphorylation signals (SLK, SSK, SRK), an Nglycosylation signal NLTH (64-67) and a possible myristolation site GLKDSR (90-95). All absolutely conserved amino acids were located within the N-terminal transmembrane helices and the loop. The sequences were also predominantly charged at the Cterminal with relatively higher proportions of lysine $(K)$, arginine $(R)$, aspartic acid (D), glutamine (Q), asparagine $(\mathrm{N})$ and glutamic acid (E). Phylogenetic analysis of the orthologues showed that the sequences clustered into three major clades constituting the various genera Plasmodium, Clostridium/Toxoplasma and Theilera/Babesia.

\section{Expression and purification of recombinant UB05}

The UB05 ORF was cloned into various expression vectors and transformed into E. coli strains for expression and purification. As expected, induction of the UB05 ORF varied depending on the vector, the host, induction conditions and whether the sub-cloned insert was truncated to code for the protein without the signal peptide or for the complete protein. Successful protein inductions with 2 mM IPTG at $42^{\circ} \mathrm{C}$ for $5 \mathrm{~h}$ or overnight could be achieved only for the truncated fragment cloned into the pEXP5NT to express a 14-kDa fusion polypeptide in BL21 (DE3)pLys (Figure 4). Low levels of expression of the truncated recom-

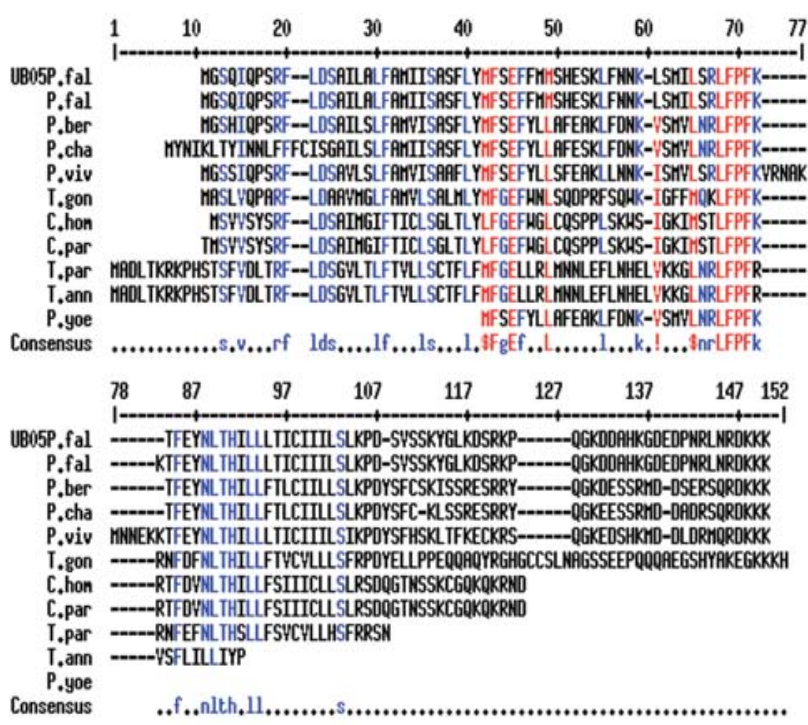

I

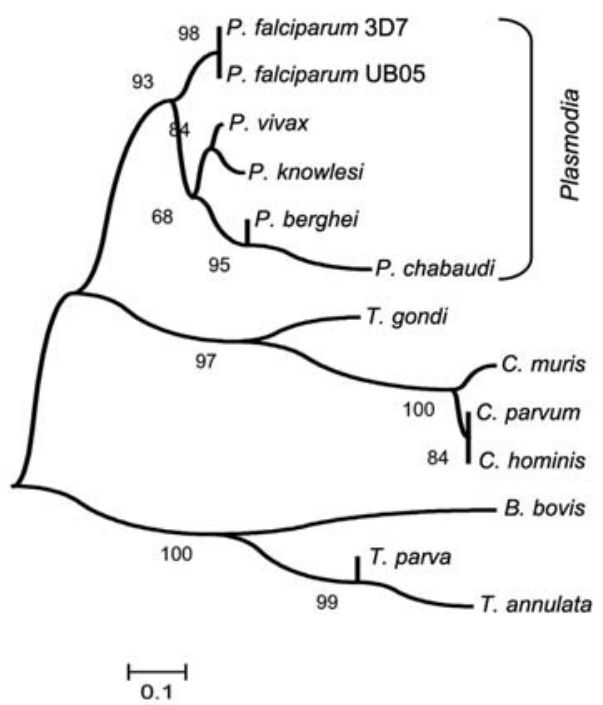

II

Figure 3 Phylogenetic relationship of UB05 homologues in infectious protozoa.

(I) Clustal alignment of UB05 homologues indicating consensus conserved amino acids. (II) The evolutionary history of UB05 protein in protozoa inferred using the Neighbour-Joining method (1). The bootstrap consensus tree inferred from 1000 replicates (2) is taken to represent the evolutionary history of the taxa analysed (2). The percentage of replicate trees in which the associated taxa clustered together in the bootstrap test (1000 replicates) are shown next to the branches. There were a total of 65 positions in the final dataset. Phylogenetic analyses were conducted in MEGA4 (4). 
A

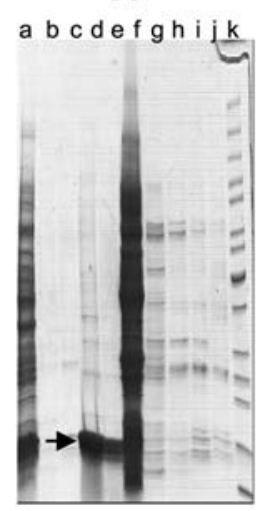

E

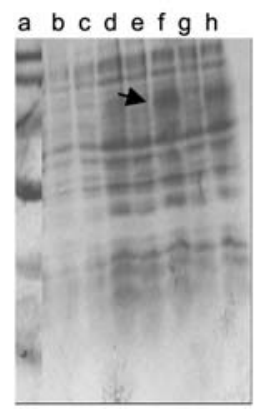

B

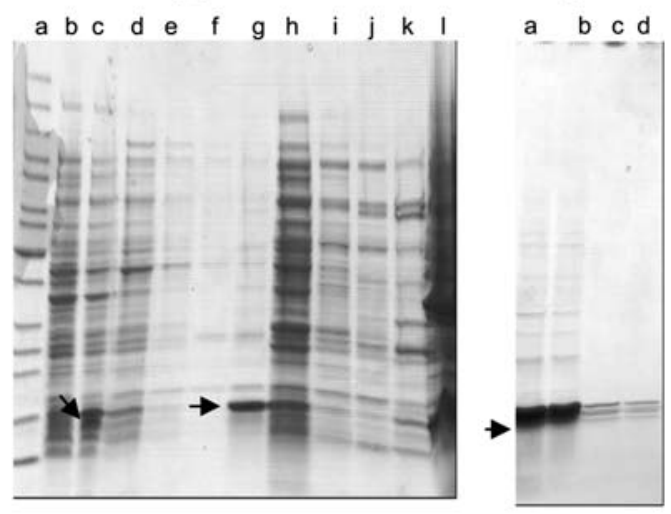

$\mathbf{F}$

G
C

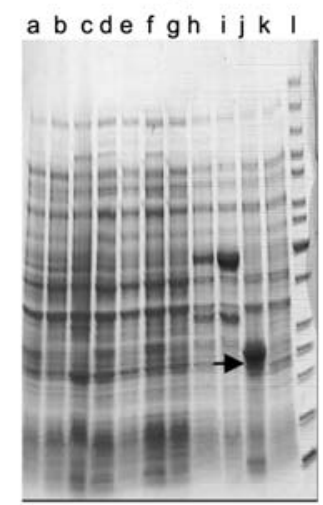

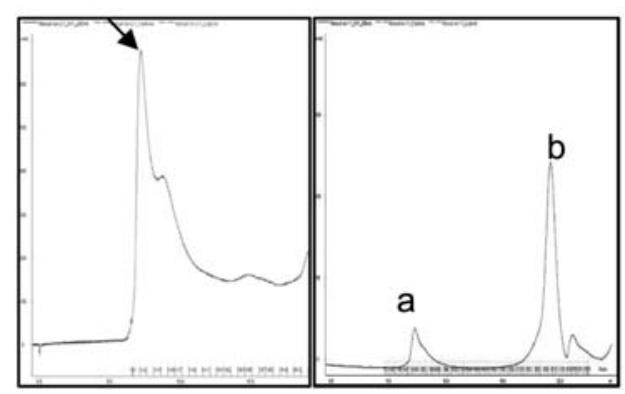

Figure 4 Expression and purification of UB05 recombinant protein.

The expressed recombinant proteins are indicated with arrowheads. (A) Overnight induction and purification gel for UB05 from pEXP5NT in BL21(De3)pLys showing an induced lysate (a), Ni-NTA washes (b, c) and eluates (d, e) under denaturing conditions and same lysate ran on Ni-NTA under native conditions $(f-j)$, molecular weight references $(k)$. (B) Purification of UB05 on Ni-NTA under denaturing conditions $(b-g)$ and native conditions ( $h-l)$ from a $5 \mathrm{~h} / 42^{\circ} \mathrm{C}$ induced BL21(DE3)pLys cell lysate. (C) Partially pure UB05 from Ni-NTA eluates ( $a$ and b) further purified on sephadex G75 gelfitration and corresponding to ARKTA chromatograms peaks $F$ with pure Ub05 proteins eluting at $\mathrm{Ga}$ and its degradation products peak $\mathrm{Hb}$. (D) Induction gel of UB05 from pETM11 in BL21; preinduced (a), induced (b); in BL21(DE3)pLys, preinduced (c), induced (d); pETM-30 in BL21, preinduced (e), induced (f), in BL21(DE3)pLys preinduced (g), induced (h and i), pETM-30 GST induction control (j), molecular weight reference (I). (E) Induction gel of UB05 protein from pBAD/thioTopo-TA in XL1Blue $(2,3)$, Novablue $(4,5)$. $(F, G)$ Spetrophotometric gelscans of recombinant UB05 separated by SDS-PAGE in the absence $(F)$ and $(G)$ in the presence of 2-mercaptoethanol. The arrows in A-E show the position of recombinant UB05 on SDS-PAGE and on F shows its position on a gelscan.

binant could also be achieved as a fusion with GST from pETM-30 in BLR, BL21 and BL21(DE3)pLys following induction with $2 \mathrm{mM}$ IPTG at cell densities $>1.0 \mathrm{OD}_{600}$ and a further $5 \mathrm{~h}$ of culture at $37^{\circ} \mathrm{C}$ (results not shown). The recombinant UB05 protein formed inclusion bodies and could be purified from induced cultures by a combination of denaturing Ni-NTA metal chelate chromatography and sephadex-G75 gel filtration in the AKTA system. Alternatively, homogeneous UB05 recombinant proteins could be obtained by eluting from SDS gel slices of fractionated eluates from His-trap chromatography. The yield of UB05 antigen after purification was calculated to be $5 \mathrm{mg}$ protein/L of culture.

\section{Amplified nucleic acid sequences of UB05 from field isolates}

All malaria infected blood samples analysed were positive for $P$. falciparum by $\mathrm{PCR}$, with two from Kumba showing mixed infections of both $P$. falciparum and $P$. malariae. Primers specific to a 285-bp truncated fragment of UB05 ORF (exons 2 and 3) successfully amplified $285 \mathrm{bp}$ and $500 \mathrm{bp}$ bands from
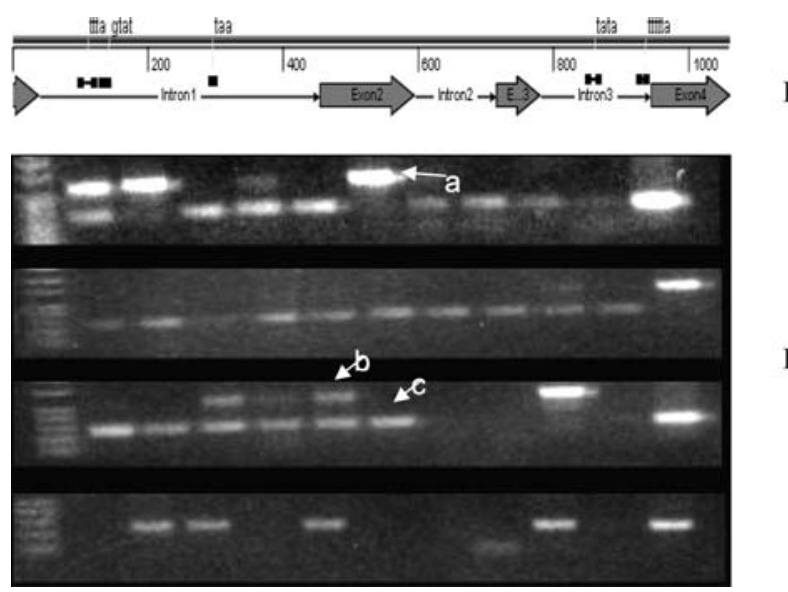

Figure 5 Description of UB05 gene.

(I) Schematic representation of the coding region of UB05 gene (PF10_0372) on P. falciparum chromosome ten showing regions of introns, exons and repeats. (II) Amplification of UB05 from DNA of field isolates of $P$. falciparum infected blood. The arrows indicate samples with a single $500 \mathrm{bp}$ amplicon (a), two amplicons of 500 and 285 bp amplicon (b), and a single amplicon of $285 \mathrm{bp}$ (c). 
genomic DNA of wild isolates (Figure 5). UB05 primers allowed for the amplification of 31 out of the 68 samples analysed from Bolifamba, representing $45.6 \%$. Of these, 17 (54.8\%) displayed both the $500 \mathrm{bp}$ and 285 bp bands. All 81 samples analysed from children in Kumba amplified as above with $60 \%$ of the samples positive for double amplicons of $285 \mathrm{bp}$ and $500 \mathrm{bp}$. Change of amplification conditions to lower or higher $\mathrm{MgCl}_{2}$ concentrations and lower template amounts did not lead to a single band. An increase in annealing temperature $>62^{\circ} \mathrm{C}$ resulted in amplification failure. Nucleic acid sequence data obtained from two isolates from Bolifamba revealed both amplicons translated into an ORF homologous to the isolated UB05 from the 3D7 $P$. falciparum cDNA library. Length polymorphisms due to indels and repeats in the introns were also seen upon further analysis of sequences of 30 wild isolates and database entries for sequenced laboratory isolates. The genomic sequences ranged from $1062 \mathrm{bp}$ in $P$. falciparum 3D7 to 1262 bp for HB3 strain. No DNA polymorphisms were observed within exons of the sequences analysed.

\section{Western blotting}

To identify the UB05 protein in field isolates, crude extracts of red blood cells infected with $P$. falciparum were separated using SDS-PAGE, transferred onto nitrocellulose and probed with plaque purified antibodies to UB05. The UB05 specific antibodies were purified from semi-immune adult human sera as described. These antibodies recognised a 38,000protein band in $P$. falciparum crude antigen extracts (Figure 6). The purified recombinant protein was immunogenic in mice and generated UB05 antibodies. To further authenticate the presence of natively translated UB05, the mouse antisera were employed in immunoblots as above. No bands could be observed with mouse pre-immune sera, demonstrating the specificity of the antibodies. The purified recombinant antigen reacted with antibodies from sera of both IC and adults, confirming its antigenicity in humans. Recombinant UB05 did not react with

\section{A}

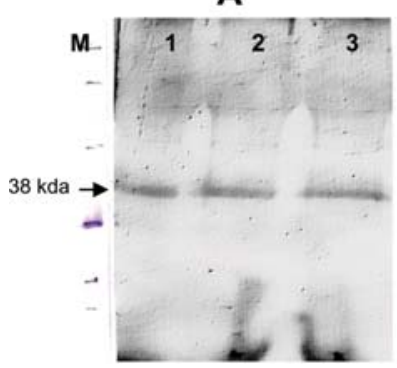

Figure 6 Antibody reactivity of UB05 in Western blots. (A) A 38-kDa band recognised by UB05 plaque purified antibodies in malaria $P$. falciparum crude blood culture antigens. (B) Reaction of UB05 recombinant protein with different sera: 1) Anti-His antibody; 2) mouse preimmune serum;3) mouse immune serum; 4) normal human serum; 5) human malaria serum and 6) no serum. M designates protein molecular weight markers. normal human sera from healthy Americans who had never visited a malaria endemic area, indicating that prior exposure to malaria parasites was necessary for immune reactivity of the antigen.

\section{Localisation of UB05 antigen}

The UB05 antibodies reacted positively in immunofluorescence assays, uniformly staining $P$. falciparum schizonts and dispersed merozoites (Figure 7).

\section{Growth inhibition studies with UB05 antibodies}

The mouse UB05 antiserum was shown to be more inhibitory $(\sim 50 \%)$ to erythrocyte invasion by $P$. falciparum 3D7 merozoites at higher titres compared to mouse pre-immune serum in preliminary reinvasion/ inhibition assays (Figure 8). Similar assays with exhaustively dialysed sera and $50 \mu \mathrm{g} / \mathrm{mL}$ of purified anti-UB05 mouse antibodies showed moderate inhibition in vitro (data not shown).

\section{Differential reactivity of UB05 with IgG}

To further study the role of UB05, we examined the serum concentrations of IgG antibodies to UB05 as a function of subject age, parasite density and febrile status. The results are presented in Figure 9. It can be seen in Figure 9A that $\lg$ concentrations to UB05 were significantly higher $(p<0.05)$ in adults compared with children. Adults in the fourth decade tended to have higher anti-UB05 IgG concentrations than those in the third decade, but the differences were not significant (results not illustrated). There was no significant difference between the antibody concentrations of the non-parasitised and parasitised adults, although the former tended to be higher. Correlation
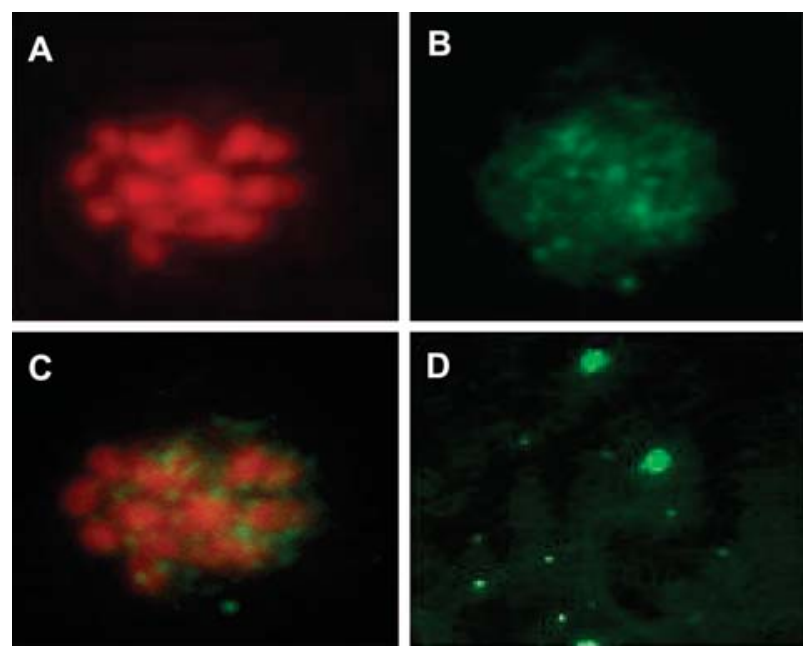

Figure 7 Localisation of UB05 antigen.

Air dried smear of $P$. falciparum F32 infected-RBC immunostained for UB05 antigen. The UB05 antigen reaction (green) and nucleus (red) were revealed with ALEXA FLOUR conjugated to chicken anti-mouse, Goat anti-chicken and ethidium bromide in that order. Each box represents a single field taken for (A) the parasite pigment; (A) nuclei; (B) UB05; (C) overlap of $A$ and $B$; (D) dispersed merozoites stained for UB05 after acetone fixation. 


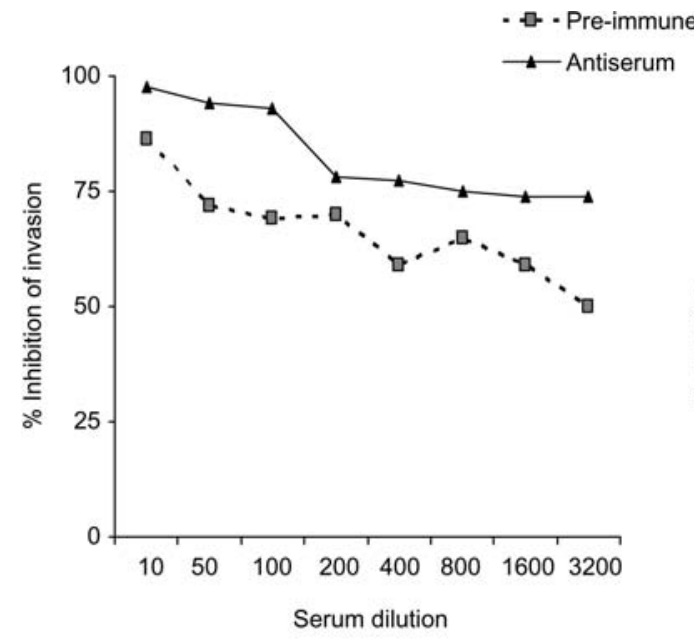

I

Inhibition assay with UB05 mouse pre-immune and immune serum

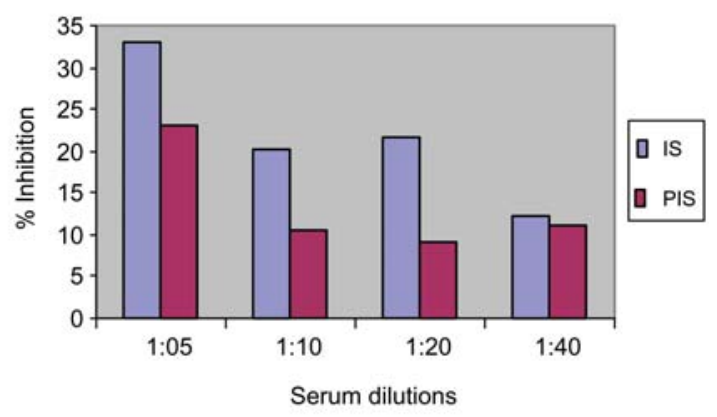

II

Figure 8 Growth inhibition studies with UB05 antibodies.

Inhibition of erythrocyte invasion by P. falciparum cultured in different dilution of mouse UB05 antiserum; (I) counted by flow cytometry; (II) by microscopy.

between antibody concentrations and parasitaemia in infected individuals showed an inverse and proportional relationship between parasite density and antiUB05 IgG antibody concentrations. Both children and adults with high anti-UB05 lgG had low or non-detectable parasitaemia (Figure 9B and C). A similar tendency was seen with the febrile status defined as a body temperature of $37.5^{\circ}$ or higher. In fact, antibody
A
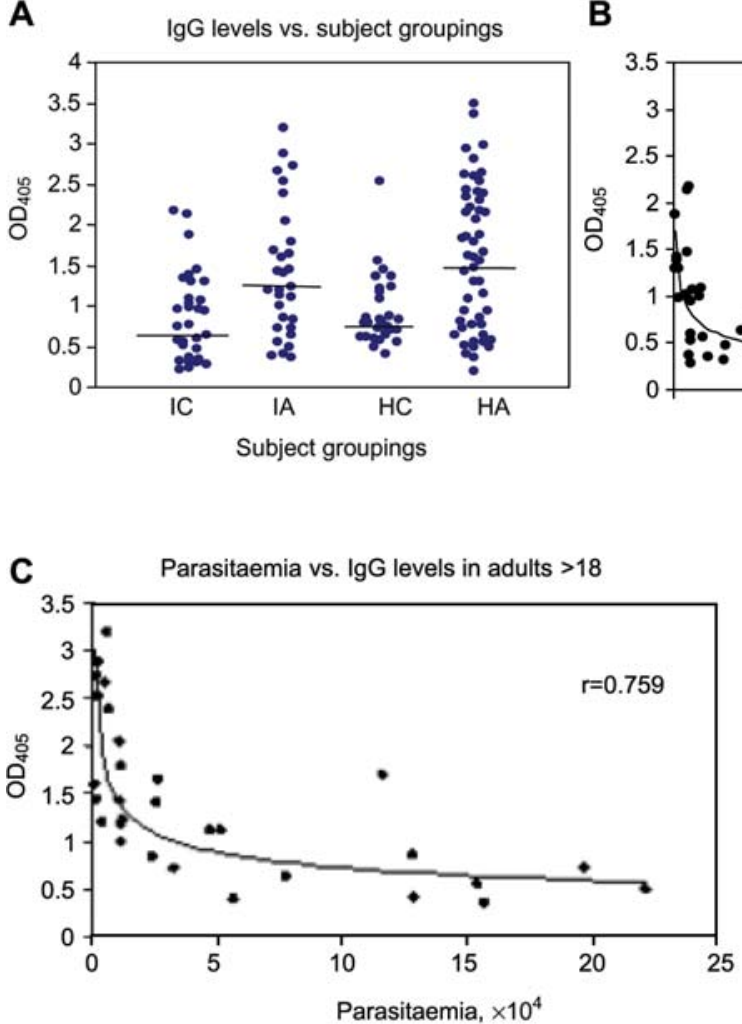

B

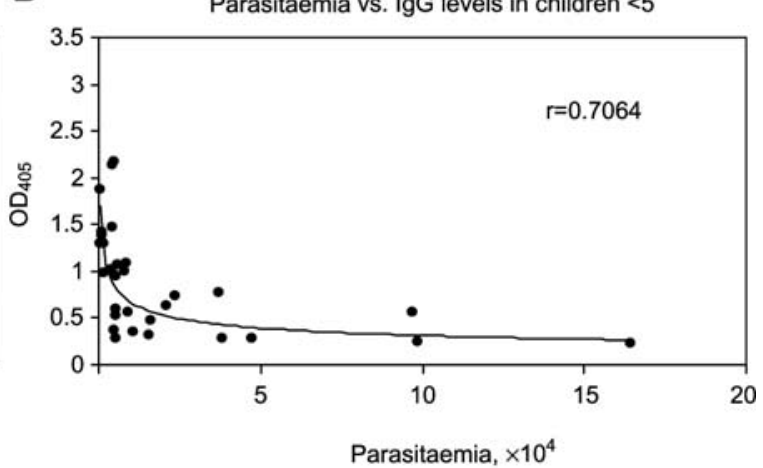

D IgG levels vs. fever status

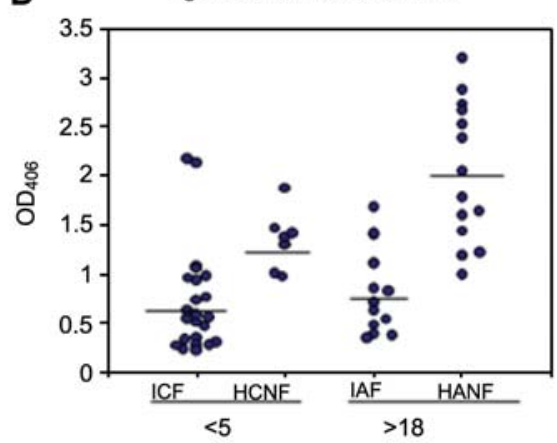

Figure 9 Differential reactivity of UB05 with IgG.

(A) Age stratified IgG responses to UB05. The children's group (IC and HC) comprise individuals of 2-5 years old and the adult group (IA and HA) individuals of 18 or older. IC, infected children, positive for $P$. falciparum; IA, infected adults positive for $P$. falciparum; $\mathrm{HC}$, healthy children negative for $P$. falciparum; $\mathrm{HA}$, healthy/semi-immune adults, negative for $P$. falciparum. The number of subjects studied per group were: $I C=29 ; I A=27 ; H C=25$ and $H A=31$. (B, C) Effect of parasitaemia on IgG levels to UB05. Parasitaemia was calculated as described under Materials and methods expressed as parasite ( $P$. falciparum) counts $/ \mathrm{mm}^{3}$. (D) Effect of malaria fever status on IgG responses $\left(O D_{406}\right)$. + The bars indicate the arithmetic mean $O D$ values of infected children with fever (ICF); healthy children without fever (HCNF); infected adults with fever (IAF) and healthy adults without fever (HANF). The number of subjects studied was as follows: ICF $=22 ; H C N F=7 ; I A F=12$ and HANF $=14$. Less than five designates children's group aged 2-5 years and $>18$ designates adult group aged 18 years or older. 
concentrations in the febrile group were significantly $(p<0.05)$ lower than in the group with normal body temperature (Figure 9D). However, we found no correlation between the anti-UB05 lgG concentrations and the presence of splenomegaly or anaemia.

\section{Discussion}

This investigation is the first to describe the involvement in host-parasite inter-play of a $P$. falciparum antigen termed UB05 (Gene Bank ID DQ235690) that was previously listed as a hypothetical protein of unknown function (8). The cDNA and deduced amino acid sequences of UB05 are identical to a portion of published $P$. falciparum 3D7 chromosome 10 genomic DNA and the corresponding open reading frame of the hypothetical protein PF10_0372, respectively (8). Clone UB05 was strongly reactive to antibodies from semi-immune adults. It consistently represented more than $50 \%$ of clones preferentially reactive to semiimmune adults following repeated screening experiments. 'Preferential reactivity' is used here to indicate that adults reacted more frequently with higher level antibodies than children (Figure 9). It is possible that the absence of reactivity to the selected HA clones by IC antibodies could be due to low titres or quality of clone specific antibodies, and not their absolute absence. However, due the dominance of UB05 in our screening panel, its identity to PF10_0372 (predicted in the TDR targets database to be in the top $93.9 \%$ of antigenic Plasmodium genes) and the coisolation of known dominant antigens, we considered it worthy of further investigation.

Searches within protein databases identified homologues of UB05 protein in other Plasmodia as well as other infectious protozoa: Cryptosporidium, Toxoplasma, Theilera and Babesia. This suggested that the isolated clone belongs to a conserved gene family in these infectious apicomplexans (Figures 2 and 3). Apart from the $P$. yoelli sequence that was truncated, homologues from all the species were predicted to have maintained conserved topological patterns, such as an $\mathrm{N}$-terminal signal leader, two transmembrane domains, sandwiching an absolutely conserved loop and a highly charged $\mathrm{C}$-terminal with multiple protein kinase $\mathrm{C}$ phosphorylation sites. It is possible that the isolated UB05 and its homologues play similar roles in these protozoan parasites. If so, this may allow for future investigation into its protective role in animal models. Proteome and transcriptome data on PlasmoDB showed maximum expression of the protein on trophozoites (27). As shown in Figure 7, UB05 antigen is also expressed in schizonts and segmented merozoites. These blood stages of Plasmodium infection remain the primary targets for vaccine development. Therefore, UB05 may be part of immunogenic antigens relevant for the development of immunity in natural infection.

The conserved topology of UB05 is not uncommon for Plasmodium proteins. In fact, 31\% (1631) of the predicted proteins of the $P$. falciparium genome have one or more transmembrane domains, and $17.3 \%$ possess putative signal peptides or signal anchors (8). Many proteins with such mophologies, for example export protein-1, have been shown to be exported to the surface or to cell organelles like the apicoplast and parasitophorous vacuole membrane, where they can be involved in physiological processes as well as immune interactions (28). Considering that our investigation also identified the immunogen, Exp-1 (UB09), this indirectly indicates that the UB05 antigen and its family of protozoan homologues could be dominant antigenic determinants in malaria. Prediction of an absolutely conserved UB05 loop (RLFPF) by the antigenic mapping software at Bcipep as a B cell epitope also suggests the antigenicity of the family. Furthermore, structural predictive analysis of the sequence following Psi-blast alignments against resolved protein structures in protein database (PDB) revealed possible folds similar to those of both intracellular and cell surface membrane proteins including translocase, ATPase subunit and receptors. These couple to the presence of three potential protein kinase $\mathrm{C}$ sites at the $\mathrm{C}$-terminus, and might implicate the molecule in some kind of intra/intercellular signalling via phosphorylation.

Primers specific to the open reading frame of UB05 were used to amplify fragments of $P$. falciparum genomic DNA isolated from the blood of infected individuals in Cameroon. These amplicons were further shown by sequencing to be identical to the isolated clone and its Pf10_0372 homologue of $P$. faciparum choromosone 10. However, the occurence of a double amplicon, despite the extreme strigency of the PCR conditions, indicated the possibility of polymorphisms. Sequencing of genomic DNA fragments of UB05 from wild isolates confirmed the size polymorphisms to be determined by indels and repeats in introns only. This further indicates that the failure of some wild isolates in PCR could have been due to indels in primer binding sequences. Sequences of wild isolates from Cameroon and five laboratory adapted isolates in PlasmoDB did not show nucleotide variation within exons, thus undermining the possibility of sequence polymorphisms (Figure 5). A desirable characteristic of some successful vaccine candidates is that they should be highly conserved and less susceptible to allelic discrimination and selection by the immune system. Our study indicated only two size alleles of UB05 and complete conservation within exon sequences in field isolates from two distinct malaria zones. Hence, the UB05 protein, in contrast to some leading polymorphic vaccine candidates, such as merozoite surface protein (MSP) - 1 and 2 and PfEMP, is relatively well conserved (29). The small size of the UB05 protein and the strong evidence of B-cell epitope conservation across infectious protozoa indicate that epitope polymorphism due to selective immune pressure in unlikely. Such immune pressure and selection are known to be responsible for genetic variation in candidate vaccine antigens and many other surface proteins amongst isolates of Plasmodium (30). 
Plaque purified human antibodies to UB05 reacted specifically with a $38-\mathrm{kDa}$ protein band (Figure 6), which was higher than the $13.8 \mathrm{kDa}$ predicted weight of the 119 aa long ORF. The sequenced UB05 cDNA was complete from start codon to a polyadenylated region, and the genomic sequences from wild isolates showed no indels within exons. Thus, the size difference may be due to abnormal migration of UB05 protein in SDS-PAGE. Such aberrant migrations in SDS-PAGE observed for other proteins have been due to aggregation, post-synthetic modifications, posttranslational cleavage, splice variants and features, such as lysine and acidic amino acid rich clusters at the c-terminal $(31,32)$. Some of these features characterise the UB05 protein and sequence and may be responsible for this abnormal PAGE migration of the protein in crude Plasmodium lysate.

A key requirement for a protective malaria antigen is that it should elicit strong immune responses in protected subjects. These responses need to be longlasting and broadly effective in controlling parasitaemia or clinical manifestations of infection. UB05 was isolated using antibodies from semi-immune adult residents of a high malaria transmission zone (Figure 1). Like most described malaria vaccine candidates, sero-epidemiological studies revealed significantly higher IgG antibody concentrations in semi-immune adults than highly susceptible children (Figure 9A and $D)$. As was the case with crude $P$. falciparum extracts, specific antibodies to UB05 increased gradually during the first two decades of life, attaining a plateau around the second and third decades (15). It is now generally accepted that lgG antibodies play a key role in the development of protective immunity to malaria that develops with age $(33,34)$, not withstanding the participation of cellular factors (35-37). The age dependent increase in IgG antibodies to UB05 is compatible with the build-up of protective immunity against malaria. However, significantly higher antiUB05 lgG titres in a few children indicate that other factors contribute to the stratification of the antibody responses in the endemic population. The inverse correlation of antibody concentrations to parasitaemia suggests that UB05 antibodies may be involved in blocking $P$. falciparum asexual stage multiplication or clearing them from circulation, although this remains to be specifically demonstrated (Figure 9B and $\mathrm{C}$ ). Preliminary assessment of anti-parasite activity in vitro in reinvasion/growth inhibition assays using rabbit anti-UB05 antibodies showed marginal levels of invasion inhibition of merozoites. This suggests that antibodies to UB05 in the endemic population may be involved in limiting parasite invasion of erythrocytes. Furthermore, the absence of fever in parasitised subjects with high concentrations of antiUB05 antibodies suggests a role for UB05 in limiting clinical malaria. The correlation between higher concentrations of antibodies against malaria with reduced parasitaemia and morbidity has been shown for a number of vaccine candidates including the serine rich antigen (SERA) and MSP3 $(38,39)$. The direct mechanism of action of these specific antibod- ies in reducing fever, even in the presence of malaria parasites, remains to be determined. However, the presence of these anti-UB05 antibodies in the absence of fever allow for the possibility that UB05 reactivity could be a marker of protective immunity against malaria.

In conclusion, the present finding suggests that UB05 is a marker of protective immunity to malaria that deserves further investigation towards UB05based vaccines.

\section{Acknowledgements}

This work was supported by grants from the International Program in the Chemical Sciences (Project IPICS/CAM 01 Uppsala University), The Microsoft Corporation (USA) and the University of Buea. We thank Professor Klavs Berzins of Stockholm University for access to facilities to conduct the growth inhibition assays.

\section{References}

1. WHO. World malaria report: WHO; 2005 4th May 2005.

2. WHO. Global malaria control and elimination; Report of a technical review; 2008.

3. Chiang PK, Bujnicki JM, Su X, Lanar DE. Malaria: therapy, genes and vaccines. Curr Mol Med 2006;6:309-26.

4. Vekemans J, Ballou WR. Plasmodium falciparum malaria vaccines in development. Expert Rev Vaccines 2008;7: 223-40.

5. Richie TL, Saul A. Progress and challenges for malaria vaccines. Nature 2002;415:694-701.

6. Greenwood B. Malaria vaccines. Evaluation and implementation. Acta Trop 2005;95:298-304.

7. Ballou WR, Arevalo-Herrera M, Carucci D, Richie TL, Corradin $G$, Diggs $C$, et al. Update on the clinical development of candidate malaria vaccines. Am J Trop Med Hyg 2004;71(2 Suppl):239-47.

8. Gardner MJ, Hall N, Fung E, White O, Berriman M, Hyman RW, et al. Genome sequence of the human malaria parasite Plasmodium falciparum. Nature 2002; 419:498-511.

9. Titanji VP, Amambua-Ngwa A, Teneng I. Molecular epidemiological approach for the identification of candidate vaccines: the case of onchocerciasis and malaria. Meeting report. 4th FASBMB Congress 2003.

10. Titanji VP, Amambua-Ngwa A, Teneng I, Tangyie E, Anong D, Akenji T. A epidemiological approach to malaria vaccine discovery: hypothesis and preliminary results. J Cameroon Acad Sci 2005;5:49-57.

11. Fandeur T, Dubois P, Gysin J, Dedet JP, da Silva LP. In vitro and in vivo studies on protective and inhibitory antibodies against Plasmodium falciparum in the Saimiri monkey. J Immunol 1984;132:432-7.

12. Lundquist $R$, Nielsen LK, Jafarshad A, Soesoe D, Christensen $L H$, Druilhe $P$, et al. Human recombinant antibodies against Plasmodium falciparum merozoite surface protein 3 cloned from peripheral blood leukocytes of individuals with immunity to malaria demonstrate antiparasitic properties. Infect Immun 2006;74:3222-31.

13. Matuschewski K, Mueller AK. Vaccines against malaria an update. Febs J 2007;274:4680-7.

14. Akenji TN, Ntonifor NN, Kimbi HK, Abongwa EL, Ching JK, Ndukum MB, et al. The epidemiology of malaria in Bolifamba, a rural community on the eastern slopes of Mount Cameroon: seasonal variation in the parasitolog- 
ical indices of transmission. Ann Trop Med Parasitol 2005;99:221-7.

15. Titanji VP, Tamu VD, Nkuo Akenji TK, Joutchop AS. Immunoglobulin $\mathrm{G}$ and subclass responses to Plasmodium falciparum antigens: a study in highly exposed Cameroonians. Clin Chem Lab Med 2002;40:937-40.

16. Ghogomu SM, Souopgui J, Pelle R, Titanji VP. Expression and characterization of Ov-47, a dominant antigen of Onchocerca volvulus. Exp Parasitol 2002;100:143-9.

17. Ljunggren EL, Nilsson D, Mattsson JG. Expressed sequence tag analysis of Sarcoptes scabiei. Parasitology 2003;127:139-45.

18. Altschul SF, Gish W, Miller W, Myers EW, Lipman DJ. Basic local alignment search tool. J Mol Biol 1990;215: 403-10.

19. Gish W, States DJ. Identification of protein coding regions by database similarity search. Nat Genet 1993;3: 266-72.

20. Marchler-Bauer A, Anderson JB, Cherukuri PF, DeWeese-Scott C, Geer LY, Gwadz M, et al. CDD: a conserved domain database for protein classification. Nucleic Acids Res 2005;33(Database issue):D192-6.

21. Marchler-Bauer A, Anderson JB, DeWeese-Scott C, Fedorova ND, Geer LY, He S, et al. CDD: a curated entrez database of conserved domain alignments. Nucleic Acids Res 2003;31:383-7.

22. Singh B, Bobogare A, Cox-Singh J, Snounou G, Abdullah MS, Rahman HA. A genus- and species-specific nested polymerase chain reaction malaria detection assay for epidemiologic studies. Am J Trop Med Hyg 1999;60: 687-92.

23. Laemmli UK. Cleavage of structural proteins during the assembly of the head of bacteriophage T4. Nature 1970;227:680-5.

24. Asubel FM. Current protocols in molecular biology: Green Publishing Associates and Whiley Interscience. 1989.

25. Wahlin $B$, Wahlgren $M$, Perlmann $H$, Berzins $K$, Bjorkman A, Patarroyo $\mathrm{ME}$, et al. Human antibodies to a $\mathrm{Mr}$ 155,000 Plasmodium falciparum antigen efficiently inhibit merozoite invasion. Proc Natl Acad Sci USA 1984;81:7912-6.

26. Bouharoun-Tayoun $\mathrm{H}$, Attanath $\mathrm{P}$, Sabchareon $A$, Chongsuphajaisiddhi T, Druilhe P. Antibodies that protect humans against Plasmodium falciparum blood stages do not on their own inhibit parasite growth and invasion in vitro, but act in cooperation with monocytes. J Exp Med 1990;172:1633-41.

27. Le Roch KG, Johnson JR, Florens L, Zhou Y, Santrosyan $A$, Grainger $M$, et al. Global analysis of transcript and protein levels across the Plasmodium falciparum life cycle. Genome Res 2004;14:2308-18.

28. Young JA, Winzeler EA. Using expression information to discover new drug and vaccine targets in the malaria parasite Plasmodium falciparum. Pharmacogenomics 2005;6:17-26.

29. Mahajan RC, Farooq U, Dubey ML, Malla N. Genetic polymorphism in Plasmodium falciparum vaccine candidate antigens. Indian J Pathol Microbiol 2005;48:42938.

30. Kidgell C, Volkman SK, Daily J, Borevitz JO, Plouffe D, Zhou $Y$, et al. A systematic map of genetic variation in Plasmodium falciparum. PLoS Pathog 2006;2:e57.

31. Curran JA, Kolakofsky D. Identification of an additional Sendai virus non-structural protein encoded by the P/C mRNA. J Gen Virol 1987;68(Pt 9):2515-9.

32. Perlmann P, Bjorkman A. Malaria research: host-parasite interactions and new developments in chemotherapy, immunology and vaccinology. Curr Opin Infect Dis 2000;13:431-43.

33. Yuen D, Leung WH, Cheung R, Hashimoto C, Ng SF, Ho $\mathrm{W}$, et al. Antigenicity and immunogenicity of the $\mathrm{N}$-terminal 33-kDa processing fragment of the Plasmodium falciparum merozoite surface protein 1, MSP1: implications for vaccine development. Vaccine 2007;25:490-9.

34. Singh S, Miura K, Zhou H, Muratova O, Keegan B, Miles $A$, et al. Immunity to recombinant plasmodium falciparum merozoite surface protein 1 (MSP1): protection in Aotus nancymai monkeys strongly correlates with antiMSP1 antibody titer and in vitro parasite-inhibitory activity. Infect Immun 2006;74:4573-80.

35. Hafalla JC, Cockburn IA, Zavala F. Protective and pathogenic roles of $\mathrm{CD} 8+\mathrm{T}$ cells during malaria infection. Parasite Immunol 2006;28:15-24.

36. Matuschewski K. Vaccine development against malaria. Curr Opin Immunol 2006;18:449-57.

37. Pombo DJ, Lawrence G, Hirunpetcharat C, Rzepczyk C, Bryden M, Cloonan N, et al. Immunity to malaria after administration of ultra-low doses of red cells infected with Plasmodium falciparum. Lancet 2002;360:610-7.

38. Okech B, Mujuzi G, Ogwal A, Shirai H, Horii T, Egwang TG. High titers of IgG antibodies against Plasmodium falciparum serine repeat antigen 5 (SERA5) are associated with protection against severe malaria in Ugandan children. Am J Trop Med Hyg 2006;74:191-7.

39. Roussilhon C, Oeuvray C, Muller-Graf C, Tall A, Rogier C, Trape JF, et al. Long-term clinical protection from falciparum malaria is strongly associated with IgG3 antibodies to merozoite surface protein 3. PLoS Med 2007; 4:e320. 